\title{
EXCITATION AND DETECTION OF VIBRATIONS OF MICROMECHANICAL STRUCTURES USING A DIELECTRIC THIN FILM
}

S. BOUWSTRA, F. R. BLOM, T. S. J. LAMMERINK, H. YNTEMA, P. SCHRAP, J. H. J. FLUITMAN and M. ELWENSPOEK

University of Twente, P.O. Box 217, 7500 AE Enschede (The Netherlands)

\begin{abstract}
A new technique is introduced for both the excitation and the detection of vibrations of micromechanical structures. This makes use of a dielectric thin film, sandwiched between lower and upper electrodes, on top of the vibrating structure. The excitation is based on electrostatic forces between the charged electrodes, causing deformation of the dielectric film and bending of the multilayer structure. The detection of the vibration is capacitive, based on the fluctuation of the capacitance due to the deformation of the dielectric film. Experimental results for a stoichiometric silicon nitride dielectric film on top of a silicon cantilever agree well with predicted values. The yield of the electrostatic excitation as well as of the capacitive detection are satisfactory.
\end{abstract}

\section{Introduction}

A growing number of sensors are based on the frequency shift of a resonating micromechanical structure. Several techniques for the excitation and detection of the vibrations of these structures have already been reported on in the literature [ 1 - 4]. In this paper, a new technique is introduced for the excitation and detection, which has specific advantages. The mechanisms are described, together with the theoretical model. We report on experiments and compare the results with the predicted behaviour.

\section{Mechanisms and theoretical model}

\section{Excitation}

A voltage applied across the dielectric film capacitor on top of a silicon cantilever causes an electrostatic attraction force between the electrodes, compressing the dielectric film in the transverse direction. The accompanying lateral expansion of the film causes bending of the multilayer, see Fig. 1. 
With a dynamic voltage of amplitude $U_{\mathrm{AC}}$ superposed on a static voltage of magnitude $U_{\mathrm{DC}}$ across the dielectric, the amplitude of the vibration at the resonance frequency at the free end of the cantilever is given by

$\delta=Q\left(\frac{3 \nu_{\mathrm{f}} \epsilon_{\mathrm{f}}\left(1-\nu_{\mathrm{s}}\right) l^{2}}{E_{\mathrm{s}}\left(1-\nu_{\mathrm{f}}\right) t_{\mathrm{f}} t_{\mathrm{s}}^{2}}\right) U_{\mathrm{DC}} U_{\mathrm{AC}}$

where $Q$ is the quality factor of the cantilever at the resonance frequency [5], $\nu_{\mathrm{f}}$ the Poisson ratio and $\epsilon_{\mathrm{f}}$ the dielectric constant of the dielectric material, $E_{\mathrm{s}}$ Young's modulus and $\nu_{\mathrm{s}}$ the Poisson ratio of the supporting layers; $t_{\mathrm{s}}$ and $t_{\mathrm{f}}$ are the thicknesses of the supporting layer and of the dielectric film respectively, and $l$ the length of the cantilever. In the derivation of expression (1), the thicknesses of the electrodes are neglected, and it is assumed that the thickness $t_{\mathrm{f}}$ of the film is small compared to the thickness $t_{\mathrm{s}}$ of the supporting layer. Further, it is assumed that the beam has a high width-to-thickness ratio and a high length-to-width ratio.

Note that the electrostatic forces not only have a dynamic component at the frequency of the applied voltage, but also a dynamic component at twice this frequency, with a magnitude proportional to $U_{\mathrm{AC}}^{2}$.

\section{Detection}

When the structure is vibrating, the upper layers are stretched. This causes a fluctuation of both the area and the thickness of the dielectric film capacitor, see Fig. 2. With a static voltage $U_{\mathbf{D C}}$ applied across the electrodes of the detection element, the alternating current $I$ charging the capacitor is found from $I=\dot{C} U_{\mathbf{D C}}$. For a detection element covering the full length of the cantilever, vibrating at its first resonance mode:

$\dot{C}=\omega \epsilon_{\mathrm{f}}\left(\frac{0.7}{1-\nu_{f}}\right)\left(\frac{w}{l}\right)\left(\frac{t_{\mathrm{s}}}{t_{f}}\right) \delta$

where $w$ is the width of the detection element, $\omega$ the radial frequency and $\delta$ the amplitude of vibration at the free end of the cantilever. The same assumptions apply as for eqn. (1). Numerical examples of yields of the
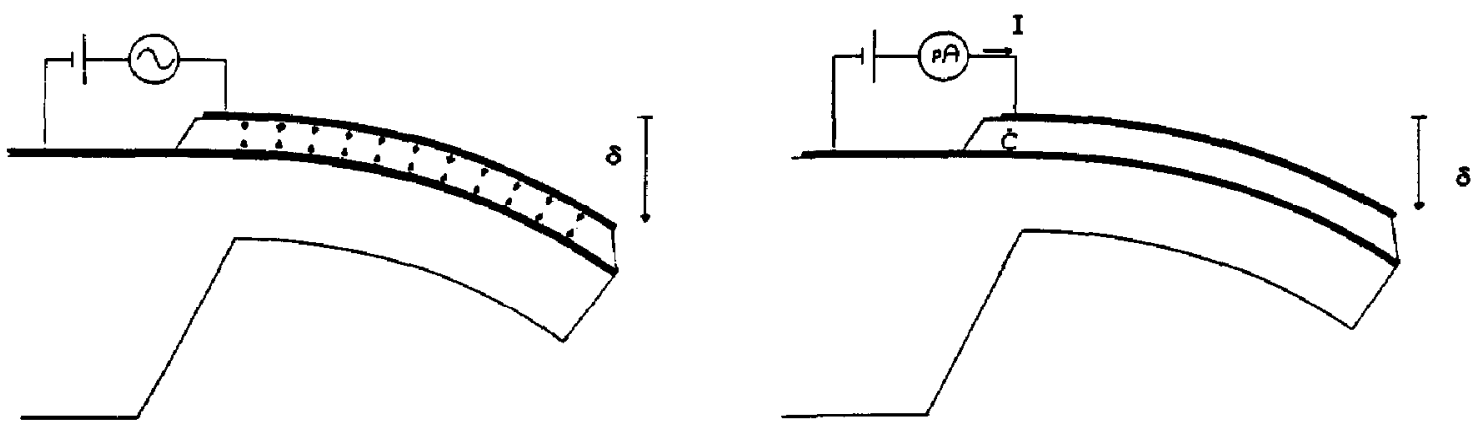

Fig. 1. Electrostatic excitation using a dielectric film.

Fig. 2. Capacitive detection using a dielectric film. 
electrostatic excitation and capacitive detection predicted by eqns. (1) and (2) are given in Table 1.

\section{Experiments}

\section{Samples}

Samples were fabricated from (100) n-type silicon wafers. First, the lower electrodes were made by CVD of boron glass and drive-in diffusion of boron $\left(R_{\square}=3 \Omega_{\square}\right)$. After removal of the glass film, stoichiometric silicon nitride was deposited by LPCVD $\left(\epsilon_{\mathrm{f}}=6.6 \times 10^{-11} \mathrm{C} \mathrm{m}^{-1} \mathrm{~V}^{-1}, t_{\mathrm{f}}=95 \mathrm{~nm}\right)$. The dielectric elements were etched on the polished side of the wafer; on the unpolished side, a mask was etched into the nitride, for micromachining at a later stage of the process sequence. Next, a $50 \mathrm{~nm}$ chromium-250 nm gold layer was evaporated for metallization. After patterning the upper electrodes, microbeams were obtained by etching the silicon substrate in a $\mathrm{KOH}-$ IPA solution. Beams of length $5 \mathrm{~mm}$, and of varying widths $(0.2 \mathrm{~mm}, 0.3$ $\mathrm{mm}, 0.4 \mathrm{~mm}, 0.5 \mathrm{~mm})$ and thicknesses $(18 \mu \mathrm{m}, 32 \mu \mathrm{m}, 41 \mu \mathrm{m})$ were realized.

\section{Testing equipment}

For the electrostatically excited microbeams, the frequency response was measured using a network analyser and a Michelson interferometer. From this the quality factor of the vibration at the first resonance mode was determined. Accurate data on the absolute amplitude of this vibration were obtained using a Mach-Zehnder heterodyne interferometer and a spectrum analyzer. The output current of the capacitive detection was measured using a lock-in amplifier, with the amplitude of the externally excited resonating beams calibrated using the Michelson interferometer.

\section{Experimental results}

The amplitude of vibration of a number of cantilevers, excited at their resonance frequency using the electrostatic excitation, was measured for a series of ranges of $U_{\mathrm{DC}}$ and $U_{\mathrm{AC}}$. A typical result of a single measurement sequence is shown in Fig. 3. The dependence of this amplitude on the static voltage $U_{\mathrm{DC}}$ showed an offset $U_{0}=5.5 \mathrm{~V}$, independent of $U_{\mathrm{AC}}$. This offset, which was also found independently in other measurements [6], is probably caused by trapped ions in the dielectric. The measured amplitude of the resonating beams proved to be proportional to $\left|U_{\mathbf{D C}}-U_{0}\right| U_{\mathbf{A C}}$ in the considered ranges, with a maximum deviation of less than $1 \mathrm{~nm}$. The reproducibility of this measurement is dependent on the resolution of the excitation frequency and on the quality factor of the vibrating cantilever. The values of the measured amplitudes corresponded to those when the beams were excited at half their resonance frequencies, with the amplitude of the resonance vibration proportional to $U_{\mathrm{AC}}^{2}$. This is a confirmation of the intended excitation mechanism. 

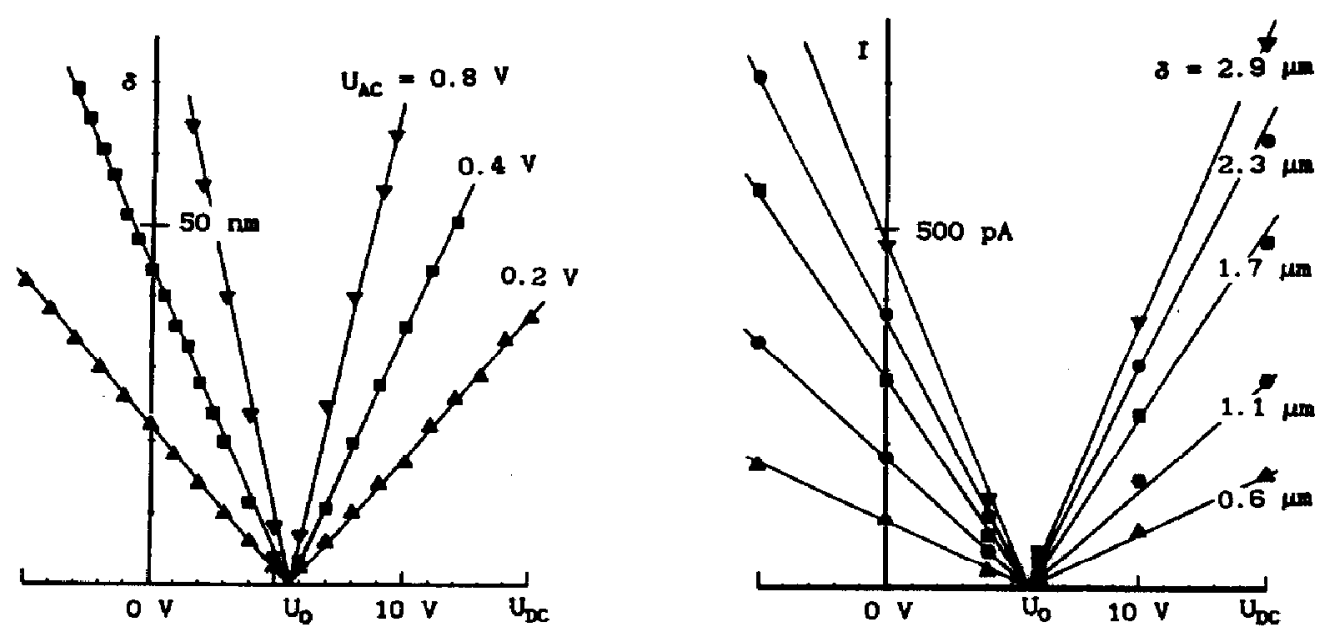

Fig. 3. Experimental results for electrostatic excitation; amplitudes of vibration $\delta$ for a range of static voltages $U_{\mathrm{DC}}$ and amplitudes of dynamic voltage $U_{\mathrm{AC}} ; t_{\mathrm{s}}=41 \mu \mathrm{m}, w=$ $530 \mu \mathrm{m}, l=5 \mathrm{~mm}$.

Fig. 4. Experimental results for capacitive detection; amplitudes of detection current $I$ for a range of static voltages $U_{\mathrm{DC}}$ and amplitudes of vibration $\delta ; t_{\mathrm{s}}=32 \mu \mathrm{m}, w=420 \mu \mathrm{m}, l=$ $5 \mathrm{~mm}$.

The output current of the capacitive detection was measured for a number of beams for a range of amplitudes of vibration and applied static voltages. A typical result is shown in Fig. 4. The output current is proportional to $\left|U_{\mathrm{DC}}-U_{0}\right| \delta$, with a maximum deviation of $10 \mathrm{pA}$. Again an offset of $5.5 \mathrm{~V}$ is observed for the applied voltage.

Measured and predicted values of the yields of the electrostatic excitation and of the capacitive detection are summarized in Table 1 for the

TABLE 1

Measured and predicted values of the yields of the electrostatic excitation and the capacitive detection; predicted values are in brackets. $\nu_{\mathrm{f}}=0.33, \epsilon_{\mathrm{f}}=6.6 \times 10^{-11} \mathrm{C} \mathrm{m}^{-1} \mathrm{~V}^{-1}$, $t_{\mathrm{f}} \doteq 95 \mathrm{~nm}, E_{\mathrm{s}}=1.69 \times 10^{11} \mathrm{~N} / \mathrm{m}^{2}, \nu_{\mathrm{s}}=0.06, l=5 \mathrm{~mm}$

\begin{tabular}{|c|c|c|c|c|c|c|c|}
\hline \multirow{2}{*}{$\frac{t_{\mathrm{s}}}{(\mu \mathrm{m})}$} & \multirow{2}{*}{$\frac{\begin{array}{l}w \\
(\mu \mathrm{m})\end{array}}{ \pm 3}$} & \multirow{2}{*}{$\frac{\begin{array}{l}\omega / 2 \pi \\
(\mathrm{Hz})\end{array}}{ \pm 1}$} & \multirow{2}{*}{$\begin{array}{l}Q \\
\pm 3 \%\end{array}$} & \multicolumn{2}{|c|}{$\begin{array}{l}\delta \\
\left(\mathrm{nm} / \mathrm{V}^{2}\right)\end{array}$} & \multicolumn{2}{|c|}{$\begin{array}{l}I \\
(\mathrm{pA} / \mu \mathrm{m} \mathrm{V})\end{array}$} \\
\hline & & & & $\pm 10 \%$ & $\pm 15 \%$ & $\pm 15 \%$ & $\pm 10 \%$ \\
\hline 41 & 230 & 2285 & 300 & 17 & [25] & & {$[20]$} \\
\hline 41 & 230 & 2292 & 330 & 15 & [29] & & [20] \\
\hline 41 & 430 & 2356 & 270 & 21 & {$[24]$} & & [38] \\
\hline 41 & 430 & 2371 & 250 & 24 & {$[21]$} & 35 & [38] \\
\hline 41 & 530 & 2341 & 220 & 17 & {$[19]$} & & {$[46]$} \\
\hline 41 & 530 & 2290 & 220 & 16 & [18] & 40 & [45] \\
\hline 32 & 220 & 1741 & 300 & 34 & [42] & & [11] \\
\hline 32 & 220 & 1845 & 290 & 28 & {$[40]$} & & [12] \\
\hline 32 & 220 & 1846 & 290 & 33 & {$[40]$} & & [12] \\
\hline 32 & 320 & 1866 & 290 & 33 & {$[40]$} & 14 & [18] \\
\hline 32 & 420 & 1937 & 300 & 32 & {$[42]$} & 29 & [24] \\
\hline 18 & 510 & 1017 & 160 & 69 & [69] & & [9] \\
\hline
\end{tabular}


samples considered. The experimental results agree well with the predicted values. Discrepancies are thought to be caused by non-uniformities in the thicknesses of the supporting layers, and by the residual stress in the highly boron-doped layer.

\section{Discussion and conclusion}

The mechanisms of the electrostatic excitation and of the capacitive detection using a dielectric thin film have been demonstrated. Experimental results and predicted values agree well. Because of trapped ions in the silicon nitride dielectric film, the application of a static voltage is not necessary for either the excitation or the detection. Although the yields of the excitation and the detection are small, they are large enough for most applications in resonant sensors.

Both yields can be increased by decreasing the thickness of the film and by applying larger voltages, or also by choosing a dielectric film material with high values of the Poisson ratio and of the dielectric constant, such as aluminium oxide. The extremely simple fabrication technology, with a wide scope of appropriate materials, geometries and etching techniques to choose from, can be made compatible with IC technology. Power consumption is low thanks to the high resistivity of the dielectric film and the high conductivity of the interconnections. This first investigation shows that this excitation and detection technique is very promising for further application in resonant sensors and in micromechanical actuators.

\section{Acknowledgements}

The authors would like to thank Rob Legtenberg for preparing the samples, and Klaas Hoen for his advise on the measurements. We would also like to thank Katrina Emmett for carefully reading the manuscript.

\section{References}

1 R. T. Howe, Resonant microsensors, Proc. 4th Int. Conf. Solid-State Sensors and Actuators (Transducers '87), Tokyo, Japan, June 2 - 5, 1987, pp. 843 - 848.

2 J. C. Greenwood, Etched silicon vibrating sensor, J, Phys. E: Sci. Instrum., 17 (1984) $650-652$.

3 J. G. Smits, H. A. C. Tilmans and T. S. J. Lammerink, Pressure dependence of resonant diaphragm pressure sensor, Proc. 3rd Int. Conf. Solid State Sensors and Actuators (Transducers '85), Philadelphia, PA, U.S.A., June 11-14, 1985, pp. 93 - 96.

4 T. S. J. Lammerink and W. Wlodarski, Integrated thermally excited resonant diaphragm pressure sensor, Proc. 3rd Int. Conf. Solid-State Sensors and Actuators (Transducers '85), Philadelphia, PA, U.S.A., June $11-14,1985$, pp. $97-100$.

5 F. R. Blom, S. Bouwstra, R. M. de Vink, J. H. J. Fluitman, M. Elwenspoek, Th. J. A. Popma and T. S. J. Lammerink, The quality factor of micromachined silicon beam resonators, Technical Digest, Eurosensors $I I$, Enschede, The Netherlands, 1988.

6 S. Bouwstra, R. Legtenberg and Th. J. A. Popma, Silicon-rich LPCVD nitride films for micromechanical applications, Technical Digest, Eurosensors II, Enschede, The Netherlands, 1988. 